\title{
DISPERSION FOR THE SCHRÖDINGER EQUATION ON NETWORKS
}

\author{
VALERIA BANICA AND LIVIU I. IGNAT
}

\begin{abstract}
In this paper we consider the Schrödinger equation on a network formed by a tree with the last generation of edges formed by infinite strips. We give an explicit description of the solution of the linear Schrödinger equation with constant coefficients. This allows us to prove dispersive estimates, which in turn are useful for solving the nonlinear Schrödinger equation. The proof extends also to the laminar case of positive step-function coefficients having a finite number of discontinuities.
\end{abstract}

\section{INTRODUCTION}

Let us first consider the linear Schrödinger equation (LSE) on $\mathbb{R}$ :

$$
\left\{\begin{array}{l}
i u_{t}+u_{x x}=0, x \in \mathbb{R}, t \in \mathbb{R} \\
u(0, x)=u_{0}(x), x \in \mathbb{R} .
\end{array}\right.
$$

The linear semigroup $e^{i t \Delta}$ has two important properties, that can be easily seen via the Fourier transform. First, the conservation of the $L^{2}$-norm:

$$
\left\|e^{i t \Delta} u_{0}\right\|_{L^{2}(\mathbb{R})}=\left\|u_{0}\right\|_{L^{2}(\mathbb{R})}
$$

and a dispersive estimate of the form:

$$
\left\|e^{i t \Delta} u_{0}\right\|_{L^{\infty}(\mathbb{R})} \leq \frac{C}{\sqrt{|t|}}\left\|u_{0}\right\|_{L^{1}(\mathbb{R})}, \quad t \neq 0 .
$$

From these two inequalities, by using the classical $T T^{*}$ argument, space-time estimates follow, known as Strichartz estimates (29, [16]):

$$
\left\|e^{i t \Delta} u_{0}\right\|_{L_{t}^{q}\left(\mathbb{R}, L_{x}^{r}(\mathbb{R})\right)} \leq C\left\|u_{0}\right\|_{L^{2}(\mathbb{R})},
$$

where $(q, r)$ are so-called admissible pairs:

$$
\frac{2}{q}+\frac{1}{r}=\frac{1}{2}, \quad 2 \leq q, r \leq \infty
$$

These dispersive estimates have been successfully applied to obtain well-posedness results for the nonlinear Schrödinger equation (see [10, [30, and the reference therein).

In this article we prove the dispersion inequality for the linear Schrödinger operator defined on a tree (bounded, connected graph without closed paths) with the external edges infinite. We assume that the tree does not contain vertices of multiplicity two, since they are irrelevant for our model. Let us notice that in this context we cannot use Fourier analysis as done on $\mathbb{R}$ for getting the dispersion inequality.

The presentation of the Laplace operator will be given in full details in the next section. Let us just say here that the Laplacian operator $\Delta_{\Gamma}$ acts as the usual

V.B. is partially supported by the ANR project "R.A.S". L. I. Ignat is partially supported by PN II-RU-TE 4/2010 and PCCE-55/2008 of CNCSIS-UEFISCSU Romania. Both authors are supported by L.E.A. project. Parts of this paper have been developed during the second author's visit to Institute Henri Poincaré, Paris. 
Laplacian on $\mathbb{R}$ on each edge, and that at vertices the Kirchhoff conditions must be fulfilled: continuity condition for the functions on the graph and transmission condition at the level of their first derivative. So our analysis will be a 1-D ramified analysis. More general coupling conditions are discussed in Section 5 .

In [18] the second author proved the same result in the case of regular trees. This means some restrictions on the shape of the trees: all the vertices of the same generation have the same number of descendants and all the edges of the same generation are of the same length. These restrictions allow to define some average functions on the edges of the same generation and to analyze some 1-D laminar Schrödinger equation (depending on the shape of the tree), where dispersion estimates were available from the first author's paper [3]. The strategy used in [18] cannot be applied in the case of a general tree and the scope of this article is to extend the class of trees where the dispersion estimate holds. In the case of a graph with a closed path, in general there exist compact supported eigenfunctions for the considered Laplace operator and then the dispersion estimate fails.

The motivation for studying thin structures comes from mesoscopic physics and nanotechnology. Mesoscopic systems are those that have some dimensions which are too small to be treated using classical physics while they are too large to be considered on the quantum level only. The quantum wires are physical systems with two dimensions reduced to a few nanometers. We refer to 23 and references therein for more details on such type of structures.

The simplest model describing conduction in quantum wires is a Hamiltonian on a planar graph, i.e. a one-dimensional object. Throughtout the paper we consider a class of idealized quantum wires, where the configuration space is a planar graph and the Hamiltonian is minus the Laplacian with Kirchhoff's boundary conditions at the vertices of the graph. This condition makes the Hamiltonian to be a self-adjoint operator. More general coupling conditions that guarantee the self-adjointness are given in 20.

The problems addressed here enter in the framework of metric graphs or networks. Those are metric spaces which can be written as the union of finitely many intervals, which are compact or $[0, \infty)$ and any two of these intervals are either disjoint or intersect only in one or both of their endpoints. Differential operators on metric graphs arise in a variety of applications. We mention some of them: carbon nano-structures [26], photonic crystals [14, high-temperature granular superconductors [1], quantum waveguides [8], free-electron theory of conjugated molecules in chemistry, quantum chaos, etc. For more details we refer the reader to review papers [23, 24, 17] and [13.

The linear and cubic Schrödinger equation on simple networks with Kirchhoff connection conditions and particular type of data has been analyzed in 9. The symmetry imposed on the initial data and the shape of the networks allow to reduce the problem to a Schrödinger equation on the half-line with appropriate boundary conditions, for which a detailed study is done by inverse scattering. Some numerical experiments are also presented in [9]. The propagation of solitons for the cubic Schrödinger equation on simple networks but with connection conditions in link with the mass and energy conservation is analyzed in [28].

The main result is the following, where by $\left\{I_{e}\right\}_{e \in E}$ we shall denote the edges of the tree.

Theorem 1.1. The solution of the linear Schrödinger equation on a tree is of the form

$$
e^{i t \Delta_{\Gamma}} \mathbf{u}_{0}(x)=\sum_{\lambda \in \mathbb{R}} \frac{a_{\lambda}}{\sqrt{|t|}} \int_{I_{\lambda}} e^{i \frac{\phi_{\lambda}(x, y)}{t}} \mathbf{u}_{0}(y) d y
$$


with $\phi_{\lambda}(x, y) \in \mathbb{R}, I_{\lambda} \in\left\{I_{e}\right\}_{e \in E}, \sum_{\lambda \in \mathbb{R}}\left|a_{\lambda}\right|<\infty$, and it satisfies the dispersion inequality

$$
\left\|e^{i t \Delta_{\Gamma}} \mathbf{u}_{0}\right\|_{L^{\infty}(\Gamma)} \leq \frac{C}{\sqrt{|t|}}\left\|\mathbf{u}_{0}\right\|_{L^{1}(\Gamma)}, \quad t \neq 0 .
$$

The proof uses the method in [3] in an appropriate way related to the ramified analysis on the tree, by recursion on the number of vertices. It consists in writing the solution in terms of the resolvent of the Laplacian, which in turn is computed in the framework of almost-periodic functions.

As mentioned before, Strichartz estimates (4) can be derived from the dispersion inequality and have been used intensively to obtain well-posedness results for the nonlinear Schrödinger equation (NSE). The arguments used in the context of NSE on $\mathbb{R}$ can also be used here to obtain the following as a typical result.

Theorem 1.2. Let $p \in(0,4)$. For any $\mathbf{u}_{0} \in L^{2}(\Gamma)$ there exists a unique solution

$$
\mathbf{u} \in C\left(\mathbb{R}, L^{2}(\Gamma)\right) \cap \bigcap_{(q, r) \text { admissible }} L_{\text {loc }}^{q}\left(\mathbb{R}, L^{r}(\Gamma)\right),
$$

of the nonlinear Schrödinger equation

$$
\begin{cases}i \mathbf{u}_{t}+\Delta_{\Gamma} \mathbf{u} \pm|\mathbf{u}|^{p} \mathbf{u}=0, & t \neq 0 \\ \mathbf{u}(0)=\mathbf{u}_{0}, & t=0 .\end{cases}
$$

Moreover, the $L^{2}(\Gamma)$-norm of $\mathbf{u}$ is conserved along the time

$$
\|\mathbf{u}(t)\|_{L^{2}(\Gamma)}=\left\|\mathbf{u}_{0}\right\|_{L^{2}(\Gamma)} .
$$

The proof is standard once the dispersion property is obtained and it follows as in [10], p. 109, Theorem 4.6.1.

With the same method we obtain the same results in the case of the Laplacian on the graph with laminar coefficients (piecewise constants, bounded between two positive constants- the details on the laminar Laplacian are given in Section 3). This might be of physical interest when the wire on a edge is composed of different pieces. Equations with variable coefficients on networks have been previously analyzed in [4] for the heat equation and in 2] for the wave equations. For clearness we prefer to treat separately the two cases even if the laminar case includes the constant coefficient case.

The paper is organized as follows. In Section 2 we introduce the Laplacian on a graph and write the systems we want to analyze. In Section 3 we present in full details the proof of Theorem 1.1. Section 4 contains the proof of the results of Theorem 1.1 in the laminar case. Some open problems are discussed in Section 5 .

\section{Notations and Preliminaries}

In this section we present some generalities about metric graphs and introduce the Laplace operator on such structure. Let $\Gamma=(V, E)$ be a graph where $V$ is a set of vertices and $E$ the set of edges. For each $v \in V$ we denote $E_{v}=\{e \in E: v \in e\}$. We assume that $\Gamma$ is a countable connected locally finite graph, i.e. the degree of each vertex $v$ of $\Gamma$ is finite: $d(v)=\left|E_{v}\right|<\infty$. The edges could be of finite length and then their ends are vertices of $V$ or they have infinite length and then we assume that each infinite edge is a ray with a single vertex belonging to $V$ (see 25] for more details on graphs with infinite edges). 
We fix an orientation of $\Gamma$ and for each oriented edge $e$, we denote by $I(e)$ the initial vertex and by $T(e)$ the terminal one. Of course in the case of infinite edges we have only initial vertices.

We identify every edge $e$ of $\Gamma$ with an interval $I_{e}$, where $I_{e}=\left[0, l_{e}\right]$ if the edge is finite and $I_{e}=[0, \infty)$ if the edge is infinite. This identification introduces a coordinate $x_{e}$ along the edge $e$. In this way $\Gamma$ is a metric space and is often named metric graph 25$]$.

Let $v$ be a vertex of $V$ and $e$ be an edge in $E_{v}$. We set for finite edges $e$

$$
j(v, e)=\left\{\begin{array}{lll}
0 & \text { if } & v=I(e), \\
l_{e} & \text { if } & v=T(e)
\end{array}\right.
$$

and

for infinite edges.

$$
j(v, e)=0, \text { if } v=I(e)
$$

We identify any function $\mathbf{u}$ on $\Gamma$ with a collection $\left\{u^{e}\right\}_{e \in E}$ of functions $u^{e}$ defined on the edges $e$ of $\Gamma$. Each $u^{e}$ can be considered as a function on the interval $I_{e}$. In fact, we use the same notation $u^{e}$ for both the function on the edge $e$ and the function on the interval $I_{e}$ identified with $e$. For a function $\mathbf{u}: \Gamma \rightarrow \mathbb{C}, \mathbf{u}=\left\{u^{e}\right\}_{e \in E}$, we denote by $f(\mathbf{u}): \Gamma \rightarrow \mathbb{C}$ the family $\left\{f\left(u^{e}\right)\right\}_{e \in E}$, where $f\left(u^{e}\right): e \rightarrow \mathbb{C}$.

A function $\mathbf{u}=\left\{u^{e}\right\}_{e \in E}$ it is continuous if and only if $u^{e}$ is continuous on $I_{e}$ for every $e \in E$, and moreover, is continuous at the vertices of $\Gamma$ :

$$
u^{e}(j(v, e))=u^{e^{\prime}}\left(j\left(v, e^{\prime}\right)\right), \quad \forall e, e^{\prime} \in E_{v} .
$$

The space $L^{p}(\Gamma), 1 \leq p<\infty$ consists of all functions $\mathbf{u}=\left\{u_{e}\right\}_{e \in E}$ on $\Gamma$ that belong to $L^{p}\left(I_{e}\right)$ for each edge $e \in E$ and

$$
\|\mathbf{u}\|_{L^{p}(\Gamma)}^{p}=\sum_{e \in E}\left\|u^{e}\right\|_{L^{p}\left(I_{e}\right)}^{p}<\infty .
$$

Similarly, the space $L^{\infty}(\Gamma)$ consists of all functions that belong to $L^{\infty}\left(I_{e}\right)$ for each edge $e \in E$ and

$$
\|\mathbf{u}\|_{L^{\infty}(\Gamma)}=\sup _{e \in E}\left\|u^{e}\right\|_{L^{\infty}\left(I_{e}\right)}<\infty .
$$

The Sobolev space $H^{m}(\Gamma), m \geq 1$ an integer, consists in all continuous functions on $\Gamma$ that belong to $H^{m}\left(I_{e}\right)$ for each $e \in E$ and

$$
\|\mathbf{u}\|_{H^{m}(\Gamma)}^{2}=\sum_{e \in E}\left\|u^{e}\right\|_{H^{m}(e)}^{2}<\infty .
$$

The above spaces are Hilbert spaces with the inner products

$$
(\mathbf{u}, \mathbf{v})_{L^{2}(\Gamma)}=\sum_{e \in E}\left(u^{e}, v^{e}\right)_{L^{2}\left(I_{e}\right)}=\sum_{e \in E} \int_{I_{e}} u^{e}(x) \overline{v^{e}}(x) d x
$$

and

$$
(\mathbf{u}, \mathbf{v})_{H^{m}(\Gamma)}=\sum_{e \in E}\left(u^{e}, v^{e}\right)_{H^{m}\left(I_{e}\right)}=\sum_{e \in E} \sum_{k=0}^{m} \int_{I_{e}} \frac{d^{k} u^{e}}{d x^{k}} \frac{\overline{d^{k} v^{e}}}{d x^{k}} d x .
$$

We now introduce the Laplace operator $\Delta_{\Gamma}$ on the graph $\Gamma$. Even if it is a standard procedure we prefer for the sake of completeness to follow 11. Consider the sesquilinear continuous form $\varphi$ on $H^{1}(\Gamma)$ defined by

$$
\varphi(\mathbf{u}, \mathbf{v})=\left(\mathbf{u}_{x}, \mathbf{v}_{x}\right)_{L^{2}(\Gamma)}=\sum_{e \in E} \int_{I_{e}} u_{x}^{e}(x) \overline{v_{x}^{e}}(x) d x .
$$


We denote by $D\left(\Delta_{\Gamma}\right)$ the set of all the functions $\mathbf{u} \in H^{1}(\Gamma)$ such that the linear map $\mathbf{v} \in H^{1}(\Gamma) \rightarrow \varphi_{\mathbf{u}}(\mathbf{v})=\varphi(\mathbf{u}, \mathbf{v})$ satisfies

$$
|\varphi(\mathbf{u}, \mathbf{v})| \leq C\|\mathbf{v}\|_{L^{2}(\Gamma)} \quad \text { for all } \mathbf{v} \in H^{1}(\Gamma) .
$$

For $\mathbf{u} \in D\left(\Delta_{\Gamma}\right)$, we can extend $\varphi_{\mathbf{u}}$ to a linear continuous mapping on $L^{2}(\Gamma)$. There is a unique element in $L^{2}(\Gamma)$ denoted by $\Delta_{\Gamma} \mathbf{u}$, such that,

$$
\varphi(\mathbf{u}, \mathbf{v})=-\left(\Delta_{\Gamma} \mathbf{u}, \mathbf{v}\right) \quad \text { for all } \mathbf{v} \in H^{1}(\Gamma) .
$$

We now define the normal exterior derivative of a function $\mathbf{u}=\left\{u^{e}\right\}_{e \in E}$ at the endpoints of the edges. For each $e \in E$ and $v$ an endpoint of $e$ we consider the normal derivative of the restriction of $\mathbf{u}$ to the edge $e$ of $E_{v}$ evaluated at $i(v, e)$ to be defined by:

$$
\frac{\partial u^{e}}{\partial n_{e}}(j(v, e))= \begin{cases}-u_{x}^{e}(0+) & \text { if } \quad j(v, e)=0 \\ u_{x}^{e}\left(l_{e}-\right) & \text { if } \quad j(v, e)=l_{e}\end{cases}
$$

With this notation it is easy to characterise $D\left(\Delta_{\Gamma}\right)$ (see [1] $)$ :

$$
D\left(\Delta_{\Gamma}\right)=\left\{\mathbf{u}=\left\{u^{e}\right\}_{e \in E} \in H^{2}(\Gamma): \sum_{e \in E_{v}} \frac{\partial u^{e}}{\partial n_{e}}(j(v, e))=0 \quad \text { for all } v \in V\right\}
$$

and

$$
\left(\Delta_{\Gamma} \mathbf{u}\right)^{e}=\left(u^{e}\right)_{x x} \quad \text { for all } e \in E, \mathbf{u} \in D\left(\Delta_{\Gamma}\right) .
$$

In other words $D\left(\Delta_{\Gamma}\right)$ is the space of all continuous functions on $\Gamma, \mathbf{u}=\left\{u^{e}\right\}_{e \in E}$, such that for every edge $e \in E, u^{e} \in H^{2}\left(I_{e}\right)$, and satisfying the following Kirchhofftype condition:

$$
\sum_{e \in E: T(e)=v} u_{x}^{e}\left(l_{e}-\right)-\sum_{e \in E: I(e)=v} u_{x}^{e}(0+)=0 \quad \text { for all } v \in V .
$$

It is easy to verify that $\left(\Delta_{\Gamma}, D\left(\Delta_{\Gamma}\right)\right)$ is a linear, unbounded, self-adjoint, dissipative operator on $L^{2}(\Gamma)$, i.e. $\Re\left(\Delta_{\Gamma} \mathbf{u}, \mathbf{u}\right)_{L^{2}(\Gamma)} \leq 0$ for all $\mathbf{u} \in D\left(\Delta_{\Gamma}\right)$.

Let us consider the LSE on $\Gamma$ :

$$
\begin{cases}i \mathbf{u}_{t}(t, x)+\Delta_{\Gamma} \mathbf{u}(t, x)=0, & x \in \Gamma, t \neq 0 \\ \mathbf{u}(0)=\mathbf{u}_{0}, & x \in \Gamma\end{cases}
$$

Using the properties of the operator $i \Delta_{\Gamma}$ we obtain as a consequence of the Hille-Yosida theorem the following well-posedness result.

Theorem 2.1. For any $\mathbf{u}_{0} \in D\left(\Delta_{\Gamma}\right)$ there exists a unique solution $\mathbf{u}(t)$ of system (9) that satisfies

$$
\mathbf{u} \in C\left(\mathbb{R}, D\left(\Delta_{\Gamma}\right)\right) \cap C^{1}\left(\mathbb{R}, L^{2}(\Gamma)\right) .
$$

Moreover, for any $\mathbf{u}_{0} \in L^{2}(\Gamma)$, there exists a unique solution $\mathbf{u} \in C\left(\mathbb{R}, L^{2}(\Gamma)\right)$ that satisfies

$$
\|\mathbf{u}(t)\|_{L^{2}(\Gamma)}=\left\|\mathbf{u}_{0}\right\|_{L^{2}(\Gamma)} \quad \text { for all } t \in \mathbb{R} .
$$

The $L^{2}(\Gamma)$-isometry property is a consequence of the fact that the operator $i \Delta_{\Gamma}$ satisfies $\Re\left(i \Delta_{\Gamma} \mathbf{u}, \mathbf{u}\right)_{L^{2}(\Gamma)}=0$ for all $\mathbf{u} \in D\left(\Delta_{\Gamma}\right)$. 
For any $\mathbf{u}_{0} \in D\left(\Delta_{\Gamma}\right)$ system (9) can be written in an explicit way as follows:

$$
\begin{cases}u^{e} \in C\left(\mathbb{R}, H^{2}\left(I_{e}\right)\right) \cap C^{1}\left(\mathbb{R}, L^{2}\left(I_{e}\right)\right), & e \in E, \\ i u_{t}^{e}(t, x)+\Delta u^{e}(t, x)=0, & x \in I_{e}, t \neq 0, \\ \text { for all } v \in V, u^{e}(t, j(v, e))=u^{e^{\prime}}\left(t, j\left(v, e^{\prime}\right)\right), & \forall e, e^{\prime} \in E_{v}, t \neq 0, \\ \sum_{e \in E: T(e)=v} u_{x}^{e}\left(t, l_{e}-\right)-\sum_{e \in E: I(e)=v} u_{x}^{e}(t, 0+)=0 & \text { for all } v \in V .\end{cases}
$$

Let us now consider the laminar case. We consider $\sigma$ a piecewise constant function on each edge of the tree such that there exist two positive constants $\sigma_{1}$ and $\sigma_{2}$ such that

$$
0<\sigma_{1}<\sigma(x)<\sigma_{2}, \quad \forall x \in I_{e}, \forall e \in E .
$$

With a similar argument as before we introduce the operator $\Delta_{\sigma, \Gamma}$ as follows

$D\left(\Delta_{\sigma, \Gamma}\right)=\left\{\mathbf{u}=\left\{u^{e}\right\}_{e \in E} \in H^{2}(\Gamma): \sum_{e \in E_{v}} \sigma(j(v, e)) \frac{\partial u^{e}}{\partial n_{e}}(j(v, e))=0 \quad\right.$ for all $\left.v \in V\right\}$ and

$$
\left(\Delta_{\sigma, \Gamma} \mathbf{u}\right)^{e}=\partial_{x}\left(\sigma(\cdot) \partial_{x}\left(u^{e}\right)\right) \text { for all } e \in E, \mathbf{u} \in D\left(\partial_{x}\left(\sigma \partial_{x}\right)\right) .
$$

$$
\begin{cases}u^{e} \in C\left(\mathbb{R}, H^{2}\left(I_{e}\right)\right) \cap C^{1}\left(\mathbb{R}, L^{2}\left(I_{e}\right)\right), & e \in E, \\ i u_{t}^{e}(t, x)+\partial_{x}\left(\sigma(x) \partial_{x} u^{e}\right)(t, x)=0, & x \in I_{e}, t \neq 0, \\ \text { for all } v \in V, u^{e}(t, j(v, e))=u^{e^{\prime}}\left(t, j\left(v, e^{\prime}\right)\right), & \forall e, e^{\prime} \in E_{v}, t \neq 0, \\ \sum_{e \in E: T(e)=v} \sigma\left(l_{e}-\right) u_{x}^{e}\left(t, l_{e}-\right)=\sum_{e \in E: I(e)=v} \sigma(0+) u_{x}^{e}(t, 0+) & \text { for all } v \in V .\end{cases}
$$

We remark that when function $\sigma$ is identically equal to one we obtain the previous system (10).

\section{The Constant COEFFicient CASE}

3.1. The description of the solution. For $\omega \geq 0$ let $R_{\omega}$ be the resolvent of the Laplacian on a tree

$$
R_{\omega} \mathbf{f}=\left(-\Delta_{\Gamma}+\omega^{2} I\right)^{-1} \mathbf{f} .
$$

We shall prove in Lemma 3.3 that $\omega R_{\omega} \mathbf{f}(x)$ can be analytically continued in a region containing the imaginary axis. Therefore we can use a spectral calculus argument to write the solution of the Schrödinger equation with initial data $\mathbf{u}_{0}$ as

$$
e^{i t \Delta_{\Gamma}} \mathbf{u}_{0}(x)=\int_{-\infty}^{\infty} e^{i t \tau^{2}} \tau R_{i \tau} \mathbf{u}_{0}(x) \frac{d \tau}{\pi} .
$$

We shall also obtain in Lemma 3.4 that the following decomposition holds

$$
\tau R_{i \tau} \mathbf{u}_{0}(x)=\sum_{\lambda \in \mathbb{R}} b_{\lambda} e^{i \tau \psi_{\lambda}(x)} \int_{I_{\lambda}} \mathbf{u}_{0}(y) e^{i \tau \beta_{\lambda} y} d y
$$

with $\psi_{\lambda}(x), \beta_{\lambda} \in \mathbb{R}, I_{\lambda} \in\left\{I_{e}\right\}_{e \in E}$ and $\sum_{\lambda \in \mathbb{R}}\left|b_{\lambda}\right|<\infty$. Then decomposition (6) is implied by (12), (13) and the fact that for $t>0$ and $r \in \mathbb{R}$

$$
\int_{-\infty}^{\infty} e^{i t \tau^{2}} e^{i \tau r} d \tau=e^{i \frac{\pi}{4}} \sqrt{\pi} \frac{e^{-\frac{r^{2}}{4 t}}}{\sqrt{t}}
$$


From (6) the dispersion estimate (7) of Theorem 1.1 follows immediately since $\sum_{\lambda \in \mathbb{R}}\left|\alpha_{\lambda}\right|<\infty$.

Above and in what follows the integration of function $\mathbf{f}=\left(f^{e}\right)_{e \in E}$ on interval $I_{e}$ means the integral of $f^{e}$ on the considered interval.

Remark 3.1. As in 3 we notice that since we can express the solution of the wave equation $\mathbf{v}_{t t}-\Delta_{\Gamma} \mathbf{v}=0$ with initial data $\left(\mathbf{v}_{0}, 0\right)$ as

$$
\mathbf{v}(t, x)=\int_{-\infty}^{\infty} e^{i t \tau} R_{i \tau} \mathbf{v}_{0}(x) i \tau \frac{d \tau}{2 \pi}
$$

the property

$$
\sup _{x \in \Gamma} \int_{-\infty}^{\infty}|\mathbf{v}(t, x)| d t \leq C\left\|\mathbf{v}_{0}\right\|_{L^{1}(\Gamma)}
$$

follows similarly. Let us mention here that the wellposedness of a class of nonlinear dispersive waves on trees, the Benjamin-Bona-Mahony equation, has been investigated in [5].

3.2. Structure of the resolvent. In order to obtain the expression of the resolvent second-order equations

$$
\left(R_{\omega} \mathbf{f}\right)^{\prime \prime}=\omega^{2} R_{\omega} \mathbf{f}-\mathbf{f}
$$

must be solved on each edge of the tree together with coupling conditions at each vertex. Then, on each edge parametrized by $I_{e}$,

$$
R_{\omega} \mathbf{f}(x)=c e^{\omega x}+\tilde{c} e^{-\omega x}+\frac{1}{2 \omega} \int_{I_{e}} \mathbf{f}(y) e^{-\omega|x-y|} d y, x \in I_{e} .
$$

Since $R_{\omega} \mathbf{f}$ belongs to $L^{2}(\Gamma)$ the coefficients $c$ 's are zero on the infinite edges $e \in \mathcal{E}$, parametrized by $[0, \infty)$. If we denote by $\mathcal{I}$ the set of internal edges, we have $2|\mathcal{I}|+|\mathcal{E}|$ coefficients. The Kirchhoff conditions of continuity of $R_{\omega} \mathbf{f}$ and of transmission of $\partial_{x} R_{\omega} \mathbf{f}$ at the vertices of the tree give the system of equations on the coefficients. We have the same number of equations as the number of unknowns. We denote $D_{\Gamma}$ the matrix of the system, whose elements are real powers of $e^{\omega}$.

$$
R_{\omega} \mathbf{f}(x)=\frac{1}{\omega \operatorname{det} D_{\Gamma}(\omega)} \sum_{\lambda=1}^{N(\Gamma)} c_{\lambda} e^{ \pm \omega \Phi_{\lambda}(x)} \int_{I_{\lambda}} \mathbf{f}(y) e^{ \pm \omega y} d y+\frac{1}{2 \omega} \int_{I_{e}} \mathbf{f}(y) e^{-\omega|x-y|} d y
$$

where $x \in I_{e}, \Phi_{\lambda}(x) \in \mathbb{R}, I_{\lambda} \in\left\{I_{e}\right\}_{e \in E}$ and $|N(\Gamma)|<\infty$. We shall prove the following proposition that will imply Lemma 3.3 and 3.4 needed for obtaining Theorem 1.1.

Proposition 3.2. Function $\operatorname{det} D_{\Gamma}(\omega)$ is lower bounded by a positive constant on a strip containing the imaginary axis:

$$
\exists c_{\Gamma}, \epsilon_{\Gamma}>0,\left|\operatorname{det} D_{\Gamma}(\omega)\right|>c_{\Gamma}, \forall \omega \in \mathbb{C},|\Re \omega|<\epsilon_{\Gamma} .
$$

Lemma 3.3. Function $\omega R_{\omega} \mathbf{f}(x)$ can be analytically continued in a region containing the imaginary axis.

Proof. The proof is an immediate consequence of decomposition (14) and of Proposition 3.2 .

Lemma 3.4. The following decomposition holds

$$
\tau R_{i \tau} \mathbf{u}_{0}(x)=\sum_{\lambda \in \mathbb{R}} b_{\lambda} e^{i \tau \psi_{\lambda}(x)} \int_{I_{\lambda}} \mathbf{u}_{0}(y) e^{i \tau \beta_{\lambda} y} d y,
$$

with $\psi_{\lambda}(x), \beta_{\lambda} \in \mathbb{R}, I_{\lambda} \in\left\{I_{e}\right\}_{e \in E}$ and $\sum_{\beta \in \mathbb{R}}\left|b_{\lambda}\right|<\infty$. 
Proof. We notice that for $\tau \in \mathbb{R}$, $\operatorname{det} D_{\Gamma}(i \tau)$ is a finite sum of powers of $e^{i \tau}$. Then, by Proposition 3.2 we are in the framework of a classical theorem in representation theory ( $\$ 29$, Cor.1 of [15]) that asserts that the inverse of $\operatorname{det} D_{\Gamma}(i \tau)$ is $\sum_{\lambda \in \mathbb{R}} d_{\lambda} e^{i \tau \lambda}$ with $\sum_{\lambda \in \mathbb{R}}\left|d_{\lambda}\right|<\infty$, and from (14) the Lemma follows.

The rest of this section is the proof of Proposition 3.2. We shall show by recursion on the number of vertices the following stronger "double" property:

$$
P(n): \quad \text { If } \Gamma \text { has } n \text { vertices, we have the property } \mathcal{P},
$$

$\mathcal{P}: \exists c_{\Gamma}, \epsilon_{\Gamma}>0, \exists 0<r_{\Gamma}<1,\left|\operatorname{det} D_{\Gamma}(\omega)\right|>c_{\Gamma},\left|\frac{\operatorname{det} \tilde{D}_{\Gamma}(\omega)}{\operatorname{det} D_{\Gamma}(\omega)}\right|<r_{\Gamma}, \forall \omega \in \mathbb{C},|\Re \omega|<\epsilon_{\Gamma}$

We have denoted by $\tilde{D}_{\Gamma}(\omega)$ the matrix of the system verified by the coefficients, if we impose that on one of the last infinite edges $l \in \mathcal{E}$ we replace in the expression of the resolvent $\tilde{c} e^{-\omega x}$ by $c e^{\omega x}$.

3.3. Proof of $P(1)$. In this case we have a star-shaped tree with $m \geq 3$ of edges. All the edges are parametrized by $[0, \infty)$. In particular $D_{\Gamma}(\omega)=D_{\Gamma}$. We shall actually prove a stronger property, which implies the property $\mathcal{P}$ for any $\epsilon_{\Gamma}>0$ :

$P(1, m)$ : If $\Gamma$ has 1 vertex and $m$ edges, $\operatorname{det} D_{\Gamma}(\omega)=m$ and $\operatorname{det} \tilde{D}_{\Gamma}(\omega)=m-2$.

The resolvent contains $\tilde{c}_{j} e^{-\omega x}, 1 \leq j \leq m$, on each of the external edges. We write matrix $D_{\Gamma_{m}}$ such that the last line is coming from Kirchhoff derivative condition, and that the other lines are coming from Kirchhoff continuity condition. So matrix $D_{\Gamma_{m}}$ can be written such that it has components 1 on the last line, and on the principal diagonal, $d_{i, i+1}=-1$ for $1 \leq j \leq m-1$, and zeros elsewhere

$$
D_{\Gamma^{m}}=\left(\begin{array}{cccccccc}
1 & -1 & & & & & & \\
& 1 & -1 & & & & & \\
& & \cdot & \cdot & & & & \\
& & & \cdot & \cdot & & & \\
& & & & \cdot & \cdot & & \\
& & & & & 1 & -1 & \\
1 & 1 & 1 & 1 & 1 & 1 & 1 & 1
\end{array}\right) \text {. }
$$

By developement with respect to the last column and $P(1, m-1)$,

$$
\operatorname{det} D_{\Gamma^{m}}=1+\operatorname{det} D_{\Gamma^{m-1}}=m .
$$

Similarly,

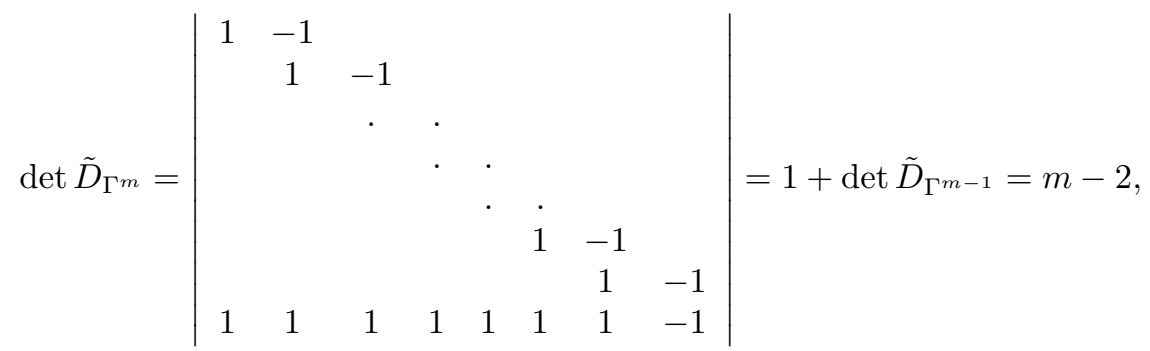

so $P(1, m)$ is proven for any $m \geq 3$ and implicitly $P(1)$. 

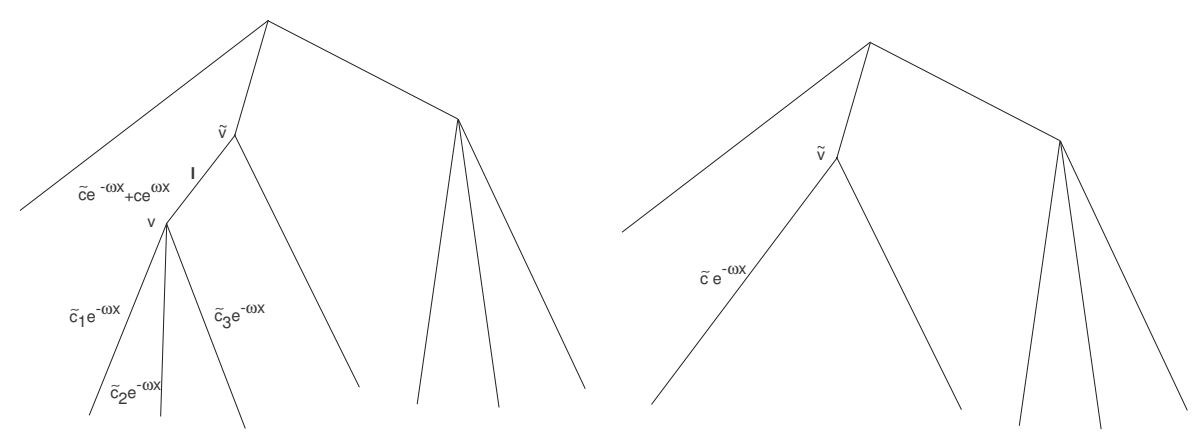

FiguRE 1. With vertex $v$ we obtain tree $\Gamma_{4}$ (left) from $\Gamma_{3}$ (right).

3.4. Proof of $P(n-1) \Rightarrow P(n)$. Any tree $\Gamma_{n}$ with $n$ vertices, $n \geq 2$, can be seen as a tree $\Gamma_{n-1}$ with $n-1$ vertices on which we add an extra-vertex. More precisely, let us consider a vertex $v$ from which there start $m \geq 2$ external infinite edges and one internal edge connecting it to the rest of the tree (see Fig. 11). Let us notice that such a choice is possible since the graph has no cycles. In particular the edge whose lower extremity is this vertex $v$ is an internal edge $l$, whose length should be denoted by $a$, and whose upper vertex we denote by $\tilde{v}$. Now we remove this vertex and transform the internal edge $l$ into an external infinite one. The new graph $\Gamma_{n-1}$ has $n-1$ vertices.

With respect to the problem on $\Gamma_{n-1}$, the resolvent on $\Gamma_{n}$ involves a new term $c e^{\omega x}$ aside from $\tilde{c} e^{-\omega x}$ on the interval edge $l$, and on the external edges emerging from the vertex $v$ it involves terms $\tilde{c}_{j} e^{-\omega x}, 1 \leq j \leq m$. We have also the Kirchhoff conditions at the vertex $v$, which give $m+1$ equations on the coefficients.

We write the square $N \times N$ matrix $D_{\Gamma_{n}}$ such that the last $m+2$ column corresponds to the unknowns $\tilde{c}, c, \tilde{c}_{1}, \ldots, \tilde{c}_{m}$. On the last line we write the Kirchhoff derivative condition at the vertex $v$, and on the $N-j$ lines, $1 \leq j \leq m$ the Kirchhoff continuity conditions at the vertex $v$. Also, on the $N-m-1$ line we write the derivative condition in the vertex $\tilde{v}$ and on the $N-m-2$ line the continuity condition in $\tilde{v}$ relating $\tilde{c}$, and now also $c$, to the others coefficients. So $D_{\Gamma_{n}}$ is a matrix obtained from the $(N-m-1) \times(N-m-1)$ matrix $D_{\Gamma_{n-1}}$ (whose last column corresponds to the unknown $\tilde{c}$ ) and from $D_{\Gamma^{m}}$ (see previous subsection) in the following way

$$
\left(D_{\Gamma_{n}}\right)_{i, j}=\left\{\begin{array}{c}
\left(D_{\Gamma_{n-1}}\right)_{i, j}, 1 \leq i, j \leq N-m-1, \\
-1,(i, j) \in\{(N-m-2, N-m),(N-m-1, N-m),(N-m, N-m+1)\}, \\
e^{\omega a},(i, j) \in\{(N-m, N-m),(N, N-m)\}, \\
e^{-\omega a},(i, j)=(N-m, N-m-1), \\
-e^{-\omega a},(i, j)=(N, N-m-1), \\
\left(D_{\Gamma^{m}}\right)_{i, j}, N-m+1 \leq i, j \leq N,
\end{array}\right.
$$




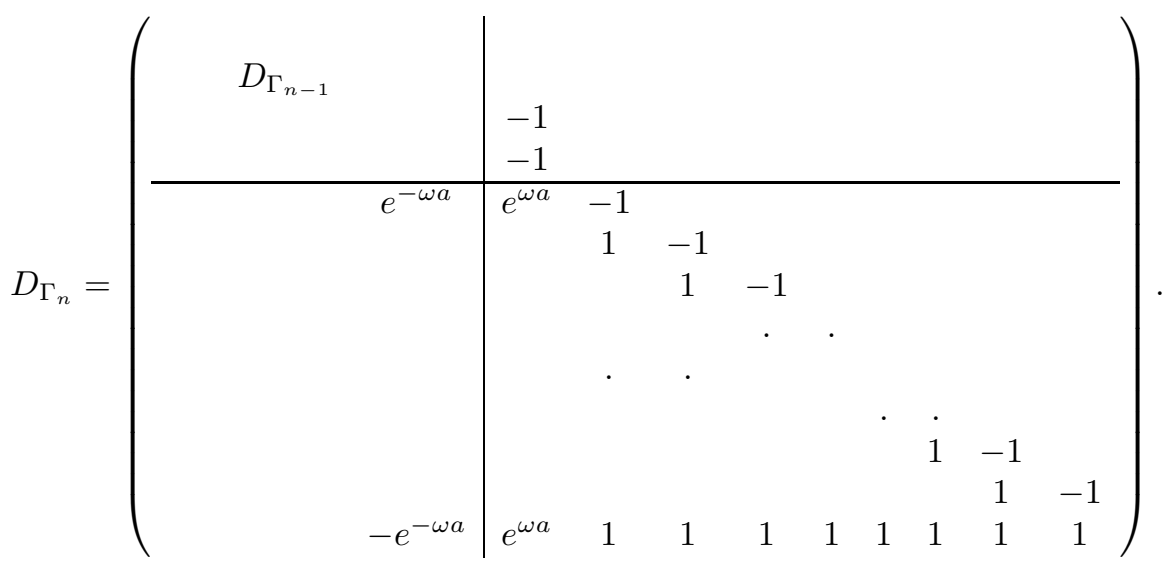

In the case $n=1$ we have that $D_{\Gamma_{1}}=D_{\Gamma^{\tilde{m}}}$ for some $\tilde{m} \geq 3$. Also, we emphasize that with the above recursion, matrix $\tilde{D}_{\Gamma_{n}}$ is obtained from $D_{\Gamma_{n}}$ by replacing its $N \times N$ element with -1 .

We develop det $D_{\Gamma_{n}}$ with respect to the last $m+1$ lines, that is as an alternated sum of determinants of $m+1 \times m+1$ minors composed from the last $m+1$ lines of $D_{\Gamma_{n}}$ times the determinant of $D_{\Gamma_{n}}$ without the lines and columns the minor is made of. The only possibility to obtain a $m+1 \times m+1$ minor composed from the last $m+1$ lines of $D_{\Gamma_{n}}$ different from zero is to choose one of the columns $N-m-1$ and $N-m$, together with all last $m$ columns. This follows from the fact that if we eliminate from $\operatorname{det} D_{\Gamma_{n}}$ both columns $N-m-1$ and $N-m$, together with $m-1$ columns among the last $m$ columns, we obtain a block-diagonal type matrix, with first diagonal block $D_{\Gamma_{n-1}}$ with its last column replaced by zeros, so its determinant vanishes. Therefore

$$
\begin{aligned}
& \operatorname{det} D_{\Gamma_{n}}=\operatorname{det} D_{\Gamma_{n-1}}\left|\begin{array}{ccccccccccc}
e^{\omega a} & -1 & & & & & & & \\
& 1 & -1 & & & & & & \\
& & 1 & -1 & & & & & \\
& & & \cdot & \cdot & & & & \\
& & & & & \cdot & \cdot & & & \\
& & & & & & \cdot & \cdot & & \\
& & & & & & 1 & -1 & \\
e^{\omega a} & 1 & 1 & 1 & 1 & 1 & 1 & 1 & 1
\end{array}\right| \\
& -\operatorname{det} \tilde{D}_{\Gamma_{n-1}}\left|\begin{array}{ccccccccccc}
e^{-\omega a} & -1 & & & & & & & \\
& 1 & -1 & & & & & & \\
& & 1 & -1 & & & & & \\
& & & \cdot & \cdot & & & & \\
& & & & & \cdot & \cdot & & & \\
& & & & & & \cdot & \cdot & & \\
& & & & & & & 1 & -1 & \\
-e^{-\omega a} & 1 & 1 & 1 & 1 & 1 & 1 & 1 & 1
\end{array}\right| .
\end{aligned}
$$

By developing with respect to the first column the $m+1 \times m+1$ minors,

$$
\begin{gathered}
\operatorname{det} D_{\Gamma_{n}}=\operatorname{det} D_{\Gamma_{n-1}}\left(e^{\omega a} \operatorname{det} D_{\Gamma^{m}}+(-1)^{m+2} e^{\omega a}(-1)^{m}\right) \\
-\operatorname{det} \tilde{D}_{\Gamma_{n-1}}\left(e^{-\omega a} \operatorname{det} D_{\Gamma^{m}}-(-1)^{m+2} e^{-\omega a}(-1)^{m}\right),
\end{gathered}
$$


so using from the previous subsection that $\operatorname{det} D_{\Gamma^{m}}=m, \operatorname{det} \tilde{D}_{\Gamma^{m}}(\omega)=m-2$, we find

$$
\begin{gathered}
\operatorname{det} D_{\Gamma_{n}}(\omega)=(m+1) e^{\omega a} \operatorname{det} D_{\Gamma_{n-1}}(\omega)-(m-1) e^{-\omega a} \operatorname{det} \tilde{D}_{\Gamma_{n-1}}(\omega) \\
=(m+1) e^{\omega a} \operatorname{det} D_{\Gamma_{n-1}}(\omega)\left(1-e^{-2 \omega a} \frac{m-1}{m+1} \frac{\operatorname{det} \tilde{D}_{\Gamma_{n-1}}(\omega)}{\operatorname{det} D_{\Gamma_{n-1}}(\omega)}\right)
\end{gathered}
$$

Now, from $P(n-1)$ we have for $|\Re \omega|$ small enough

$$
1-e^{-2 \omega a} \frac{m-1}{m+1} \frac{\operatorname{det} \tilde{D}_{\Gamma_{n-1}}(\omega)}{\operatorname{det} D_{\Gamma_{n-1}}(\omega)}>c_{0}>0 .
$$

Also, $P(n-1)$ gives us the existence of two positive constants $c_{\Gamma_{n-1}}$ and $\epsilon_{\Gamma_{n-1}}$ such that $\left|\operatorname{det} D_{\Gamma_{n-1}}(\omega)\right|>c_{\Gamma_{n-1}}, \forall \omega \in \mathbb{C},|\Re \omega|<\epsilon_{\Gamma_{n-1}}$, so eventually we get

$$
\exists c_{\Gamma_{n}}, \epsilon_{\Gamma_{n}}>0,\left|\operatorname{det} D_{\Gamma_{n}}(\omega)\right|>c_{\Gamma_{n}}, \forall \omega \in \mathbb{C},|\Re \omega|<\epsilon_{\Gamma_{n}},
$$

and the first part of property $\mathcal{P}$ is proved for $P(n)$.

In a similar way we get

$$
\operatorname{det} \tilde{D}_{\Gamma_{n}}(\omega)=(m-1) e^{\omega a} \operatorname{det} D_{\Gamma_{n-1}}(\omega)-(m-3) e^{-\omega a} \operatorname{det} \tilde{D}_{\Gamma_{n-1}}(\omega),
$$

so

$$
\frac{\operatorname{det} \tilde{D}_{\Gamma_{n}}(\omega)}{\operatorname{det} D_{\Gamma_{n}}(\omega)}=\frac{\frac{m-1}{m+1}-\frac{m-3}{m+1} e^{-2 \omega a} \frac{\operatorname{det} \tilde{D}_{\Gamma_{n-1}}(\omega)}{\operatorname{det} D_{\Gamma_{n-1}}(\omega)}}{1-\frac{m-1}{m+1} e^{-2 \omega a} \frac{\operatorname{det} \tilde{D}_{\Gamma_{n-1}}(\omega)}{\operatorname{det} D_{\Gamma_{n-1}}(\omega)}} .
$$

Thus we also get the second part of $\mathcal{P}$ for $P(n)$ since

$$
\left|\frac{\frac{m-1}{m+1}-\frac{m-3}{m+1} z}{1-\frac{m-1}{m+1} z}\right|<1 \Longleftrightarrow 0<(m-2)\left(|z|^{2}-1\right)+2(m-1)(1-\Re z) .
$$

\section{The LAMinar COEFFicient CASE}

For $\omega \geq 0$ let $R_{\omega}$ be the resolvent of the operator $\Delta_{\sigma, \Gamma}$ defined in Section 2

$$
R_{\omega} \mathbf{f}=\left(-\Delta_{\sigma, \Gamma}+\omega^{2} I\right)^{-1} \mathbf{f} .
$$

We shall proceed as in the previous section, and the main point will be the proof of Proposition 3.2 in the laminar coefficient case. On each side of the edge, parametrized by $x \in I \subset \mathbb{R}$, where the coefficient in the laminar Laplacian is $\sigma(x)=\frac{1}{b^{2}}$, the resolvent writes

$$
R_{\omega} \mathbf{f}(x)=c e^{\omega b x}+\tilde{c} e^{-\omega b x}+\frac{1}{2 \omega} \int_{I} \mathbf{f}(y) e^{-\omega b|x-y|} d y, x \in I .
$$

As in the previous section, and using the same notations, we shall show by recursion on the number of vertices property $P(n)$ which leads to the dispersion estimate.

4.1. Proof of $P(1)$. We prove property $P(1)$ on a star-shaped tree by recursion on the number of discontinuities in the laminar structure:

$P(1, p)$ : If $\Gamma$ has 1 vertex and $p$ discontinuities along its edges we have property $\mathcal{P}$.

We denote by $m \geq 3$ the number of edges. 
We start with $P(1,0)$. We denote by $\frac{1}{b_{j}^{2}}, 1 \leq j \leq m$ the coefficients of the laminar Laplacian on each edge. The resolvent contains the terms $\tilde{c}_{j} e^{-\omega b_{j} x}, 1 \leq j \leq m$ (and $c_{m} e^{\omega b_{3} x}$ on the last edge for the computation of $\left.\tilde{D}_{\Gamma}\right)$ on each edge. We have

$$
D_{\Gamma^{m}, 0}=\left(\begin{array}{ccccccccc}
1 & -1 & & & & & & \\
& 1 & -1 & & & & & \\
& & \cdot & . & & & & \\
& & & \cdot & . & & & \\
& & & & \cdot & . & & \\
& & & & & 1 & -1 & \\
\frac{1}{b_{1}} & \frac{1}{b_{2}} & \frac{1}{b_{3}} & . . & . . & \frac{1}{b_{m-2}} & \frac{1}{b_{m-1}} & \frac{1}{b_{m}}
\end{array}\right) .
$$

By developing with respect to the last column,

$$
\operatorname{det} D_{\Gamma^{m, 0}}=\frac{1}{b_{m}}+\operatorname{det} D_{\Gamma^{m-1,0}}=\sum_{j=1}^{m} \frac{1}{b_{j}} .
$$

Similarly we obtain

$$
\operatorname{det} \tilde{D}_{\Gamma^{m, 0}}=\sum_{j=1}^{m-1} \frac{1}{b_{j}}-\frac{1}{b_{m}},
$$

so the property $\mathcal{P}$ follows immediately.

Now we shall prove that $P(1, p-1)$ implies $P(1, p)$. Without loss of generality we can suppose that on the last $m$-th edge of $\Gamma^{m, p}$ there is at least one discontinuity. We denote by $x_{f}$ the last discontinuity point on this edge, and by $x_{i}$ the previous discontinuity if there is one, or $x_{i}=0$ otherwise. We denote $a$ the length $x_{f}-x_{i}$, by $\frac{1}{b_{i}^{2}}$ the coefficient of the laminar Laplacian on $\left(x_{i}, x_{f}\right)$ and by $\frac{1}{b_{f}^{2}}$ the coefficient of the laminar Laplacian on $\left(x_{f}, \infty\right)$. We call $\Gamma^{m, p-1}$ the graph obtained from $\Gamma^{m, p}$ by removing the last discontinuity $x_{f}$ on the last edge, and we extend the laminar Laplacian on it on $\left[x_{f}, \infty\right)$ by $\frac{1}{b_{i}^{2}}$.

With respect to the problem on $\Gamma^{m, p-1}$, in the expression of the resolvent we have on $\left[x_{i}, x_{f}\right]$ aside from the term $\tilde{c}_{i} e^{-\omega b_{i} x}$, the extra term $c_{i} e^{\omega b_{i} x}$, and on $\left[x_{f}, \infty\right)$ a term $\tilde{c}_{f} e^{-\omega b_{f} x}$. Also, there are two connection conditions at the new discontinuity point $x_{f}$. We write the matrix $D_{\Gamma^{m, p}}$ such that the last three columns correspond to the unknowns $\left(\tilde{c}_{i}, c_{i}, \tilde{c}_{f}\right)$, the last two lines come from the connection condition at $x_{f}$, and the previous last two lines come from the connection condition at $x_{i}$, if $x_{i}$ is a discontinuity point, and from the Kirchhoff conditions concerning $c_{i}, \tilde{c}_{i}$ if it is the vertex.

We have

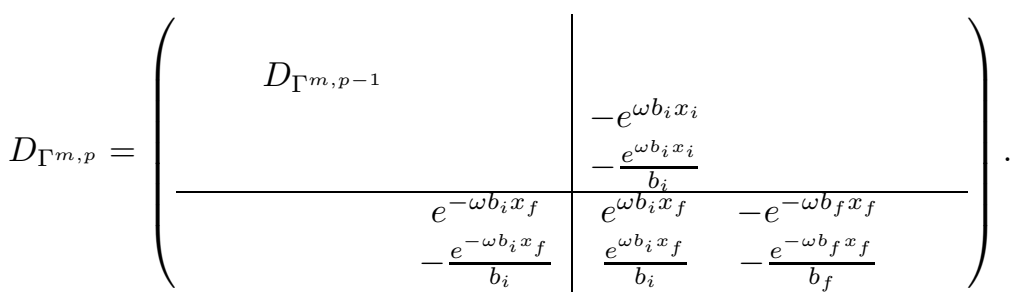

By developing with respect to the last two lines,

$$
\begin{gathered}
\operatorname{det} D_{\Gamma^{m, p}}=e^{\omega b_{i} x_{f}} e^{-\omega b_{f} x_{f}}\left(\frac{1}{b_{f}}+\frac{1}{b_{i}}\right) \operatorname{det} D_{\Gamma^{m, p-1}}-e^{-\omega b_{i} x_{f}} e^{-\omega b_{f} x_{f}}\left(\frac{1}{b_{f}}-\frac{1}{b_{i}}\right) \operatorname{det} \tilde{D}_{\Gamma^{m, p-1}} \\
=e^{\omega b_{i} x_{f}} e^{-\omega b_{f} x_{f}}\left(\frac{1}{b_{f}}+\frac{1}{b_{i}}\right) \operatorname{det} D_{\Gamma^{m, p-1}}\left(1-e^{-2 \omega b_{i} x_{f}} \frac{\frac{1}{b_{f}}-\frac{1}{b_{i}}}{\frac{1}{b_{f}}+\frac{1}{b_{i}}} \frac{\operatorname{det} \tilde{D}_{\Gamma^{m, p-1}}}{\operatorname{det} D_{\Gamma^{m, p-1}}}\right) .
\end{gathered}
$$


Therefore $P(1, p-1)$ implies the first part of property $P(1, p)$. In a similar way we compute

$\operatorname{det} \tilde{D}_{\Gamma^{m, p}}=e^{\omega b_{i} x_{f}} e^{\omega b_{f} x_{f}}\left(-\frac{1}{b_{f}}+\frac{1}{b_{i}}\right) \operatorname{det} D_{\Gamma^{m, p-1}}-e^{-\omega b_{i} x_{f}} e^{\omega b_{f} x_{f}}\left(-\frac{1}{b_{f}}-\frac{1}{b_{i}}\right) \operatorname{det} \tilde{D}_{\Gamma^{m, p-1}}$, So

$$
\frac{\operatorname{det} \tilde{D}_{\Gamma^{m, p}}}{\operatorname{det} D_{\Gamma^{m, p}}}=e^{2 \omega b_{f} x_{f}} \frac{\frac{-\frac{1}{b_{f}}+\frac{1}{b_{i}}}{\frac{1}{b_{f}}+\frac{1}{b_{i}}}+e^{-2 \omega b_{i} x_{f}} \frac{\operatorname{det} \tilde{D}_{\Gamma^{m, p-1}}}{\operatorname{det} D_{\Gamma^{m, p-1}}}}{1+e^{-2 \omega b_{i} x_{f}} \frac{-\frac{1}{b_{f}}+\frac{1}{b_{i}}}{\frac{1}{b_{f}}+\frac{1}{b_{i}}} e^{-2 \omega b_{i} x_{f} \frac{\operatorname{det} \tilde{D}_{\Gamma^{m, p-1}}}{\operatorname{det} D_{\Gamma^{m, p-1}}}}},
$$

and the second part of $P(1, p)$ follows also from $P(1, p-1)$ by noticing that for $a$ real

$$
\left|\frac{a+z}{1+a z}\right|<1 \Longleftrightarrow 0<\left(1-a^{2}\right)\left(1-|z|^{2}\right) .
$$

In conclusion $P(1)$ is proved.

4.2. Proof of $P(n-1) \Rightarrow P(n)$. Let us consider a vertex $v$ from which there start $m \geq 2$ external infinite edges and one internal edge connecting it to the rest of the graph. The proof of $P(1, p-1) \Rightarrow P(1, p)$, of eliminating discontinuities on infinite edges also works for trees with $n \geq 1$ vertices. So it is enough to prove $P(n)$ for a graph $\Gamma_{n}$ with no discontinuities on the infinite edges emanating from vertex $v$. We call $\frac{1}{b_{j}^{2}}, 1 \leq j \leq m$ the coefficients of the laminar Laplacian on the $m$ edges emerging from $v$. With the notations of the previous subsection and from $\$ 3.4$ we have

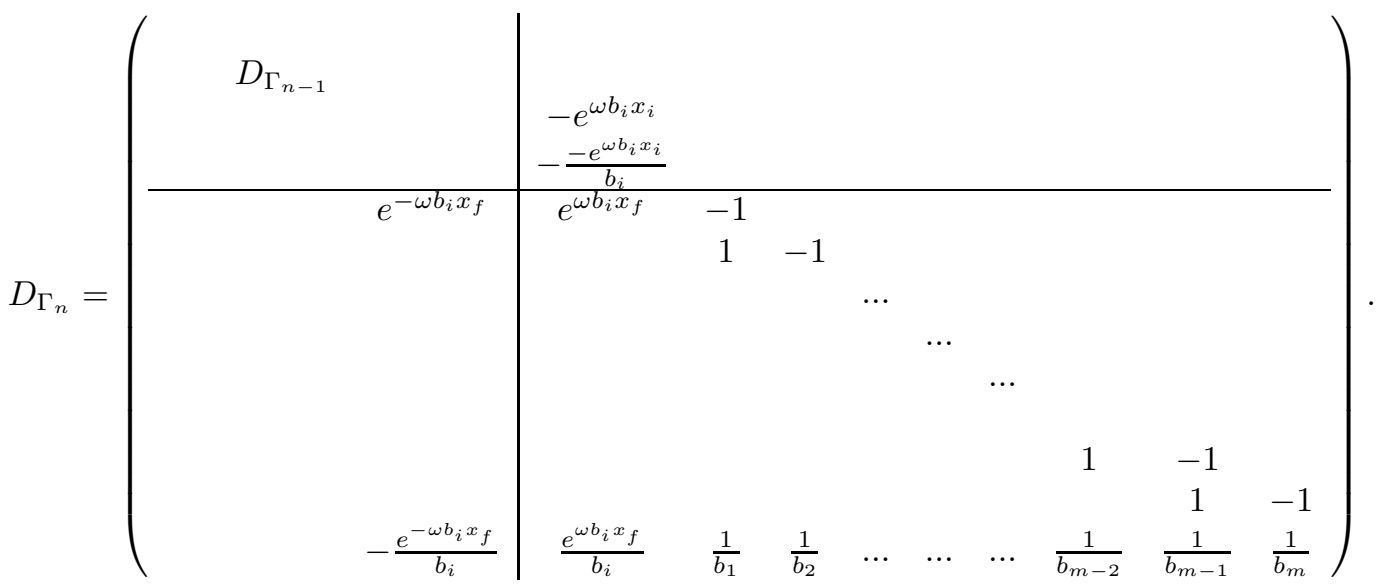

By developing with respect to the first column the $m+1 \times m+1$ minors,

$$
\begin{gathered}
\operatorname{det} D_{\Gamma_{n}}=\operatorname{det} D_{\Gamma_{n-1}}\left(e^{\omega b_{i} x_{f}} \operatorname{det} D_{\Gamma^{m}}+(-1)^{m+2} \frac{e^{\omega b_{i} x_{f}}}{b_{i}}(-1)^{m}\right) \\
-\operatorname{det} \tilde{D}_{\Gamma_{n-1}}\left(e^{-\omega b_{i} x_{f}} \operatorname{det} D_{\Gamma^{m}}-(-1)^{m+2} \frac{e^{-\omega b_{i} x_{f}}}{b_{i}}(-1)^{m}\right)
\end{gathered}
$$

so using from the previous subsection the values of $\operatorname{det} D_{\Gamma^{m}}$ and $\operatorname{det} \tilde{D}_{\Gamma^{m}}$,

$$
\begin{gathered}
\operatorname{det} D_{\Gamma_{n}}(\omega)=e^{\omega b_{i} x_{f}}\left(\sum_{j=1}^{m} \frac{1}{b_{j}}+\frac{1}{b_{i}}\right) \operatorname{det} D_{\Gamma_{n-1}}-e^{-\omega b_{i} x_{f}}\left(\sum_{j=1}^{m} \frac{1}{b_{j}}-\frac{1}{b_{i}}\right) \operatorname{det} \tilde{D}_{\Gamma_{n-1}} \\
=e^{\omega b_{i} x_{f}}\left(\sum_{j=1}^{m} \frac{1}{b_{j}}+\frac{1}{b_{i}}\right) \operatorname{det} D_{\Gamma_{n-1}}\left(1-e^{-2 \omega b_{i} x_{f}} \frac{\sum_{j=1}^{m} \frac{1}{b_{j}}-\frac{1}{b_{i}}}{\sum_{j=1}^{m} \frac{1}{b_{j}}+\frac{1}{b_{i}}} \frac{\operatorname{det} \tilde{D}_{\Gamma_{n-1}}}{\operatorname{det} D_{\Gamma_{n-1}}}\right) .
\end{gathered}
$$


Therefore, using $P(n-1)$ we get the first part of $P(n)$. In a similar way we get

$\operatorname{det} \tilde{D}_{\Gamma_{n}}(\omega)=e^{\omega b_{i} x_{f}}\left(\sum_{j=1}^{m-1} \frac{1}{b_{j}}-\frac{1}{b_{m}}+\frac{1}{b_{i}}\right) \operatorname{det} D_{\Gamma_{n-1}}-e^{-\omega b_{i} x_{f}}\left(\sum_{j=1}^{m-1} \frac{1}{b_{j}}-\frac{1}{b_{m}}-\frac{1}{b_{i}}\right) \operatorname{det} \tilde{D}_{\Gamma_{n-1}}$,

so

$$
\frac{\operatorname{det} \tilde{D}_{\Gamma_{n}}}{\operatorname{det} D_{\Gamma_{n}}}=\frac{\frac{\sum_{j=1}^{m-1} \frac{1}{b_{j}}-\frac{1}{b_{m}}+\frac{1}{b_{i}}}{\sum_{j=1}^{m} \frac{1}{b_{j}}+\frac{1}{b_{i}}}-e^{-2 \omega b_{i} x_{f}} \frac{\sum_{j=1}^{m-1} \frac{1}{b_{j}}-\frac{1}{b_{m}}-\frac{1}{b_{i}}}{\sum_{j=1}^{m} \frac{1}{b_{j}}+\frac{1}{b_{i}}} \frac{\operatorname{det} \tilde{D}_{\Gamma_{n-1}}}{\operatorname{det} D_{\Gamma_{n-1}}}}{1-e^{-2 \omega b_{i} x_{f}}} .
$$

Since for $a, b, c$ real

$$
\left|\frac{\frac{a-b+c}{a+b+c}-\frac{a-b-c}{a+b+c} z}{1-\frac{a+b-c}{a+b+c} z}\right| \leq 1 \Longleftrightarrow 0<a b\left(\Im^{2} z+(\Re z-1)^{2}\right)+b c\left(1-|z|^{2}\right),
$$

the second part of $P(n)$ follows, completing the proof in the laminar case.

\section{Open Problems}

In this paper we have analyzed the dispersive properties for the linear Schrödinger equation on trees. We have assumed that the coupling is given by the classical Kirchhoff's conditions. However there are other coupling conditions (see [20]) which allow to define a "Laplace" operator on a metric graph. To be more precise, let us consider the operator $H$ that acts on functions on the graph $\Gamma$ as the second derivative $\frac{d^{2}}{d x^{2}}$, and its domain consists in all functions $f$ that belong to the Sobolev space $H^{2}(e)$ on each edge $e$ of $\Gamma$ and satisfy the following boundary condition at the vertices:

$$
A(v) \mathbf{f}(v)+B(v) \mathbf{f}^{\prime}(v)=0 \text { for each vertex } v .
$$

Here $\mathbf{f}(v)$ and $\mathbf{f}^{\prime}(v)$ are correspondingly the vector of values of $f$ at $v$ attained from directions of different edges converging at $v$ and the vector of derivatives at $v$ in the outgoing directions. For each vertex $v$ of the tree we assume that matrices $A(v)$ and $B(v)$ are of size $d(v)$ and satisfy the following two conditions

(1) the joint matrix $(A(v), B(v))$ has maximal rank, i.e. $d(v)$,

(2) $A(v) B(v)^{T}=B(v) A(v)^{T}$.

Under those assumptions it has been proved in 20] that the considered operator, denoted by $\Delta(A, B)$, is self-adjoint. The case considered in this paper, the Kirchhoff coupling, corresponds to the matrices

$$
A(v)=\left(\begin{array}{cccccc}
1 & -1 & 0 & \ldots & 0 & 0 \\
0 & 1 & -1 & \ldots & 0 & 0 \\
0 & 0 & 1 & \ldots & 0 & 0 \\
\vdots & \vdots & \vdots & & \vdots & \vdots \\
0 & 0 & 0 & \vdots & 1 & -1 \\
0 & 0 & 0 & \vdots & 0 & 0
\end{array}\right), B(v)=\left(\begin{array}{cccccc}
0 & 0 & 0 & \ldots & 0 & 0 \\
0 & 0 & 0 & \ldots & 0 & 0 \\
0 & 0 & 0 & \ldots & 0 & 0 \\
\vdots & \vdots & \vdots & & \vdots & \vdots \\
0 & 0 & 0 & \ldots & 0 & 0 \\
1 & 1 & 1 & \ldots & 1 & 1
\end{array}\right) .
$$

More examples of matrices satisfying the above conditions are given in [20, 19 .

The existence of the dispersive properties for the solutions of the Schrödinger on a graph under general coupling conditions on the vertices $i u_{t}+\Delta_{\Gamma}(A, B) u=0$ is mainly an open problem. The resolvent formula obtained in [19] and [22] in terms of the coupling matrices $A$ and $B$ might help to understand the general problem. In the same papers there are also some combinatorial formulations of the resolvent in 
terms of walks on graphs. Such combinational aspects could clarify if the dispersion is possible only on trees or there are graphs (with some of the edges infinite) with suitable couplings where the dispersion is still true.

It is expected that other results on the Schrödinger equation on $\mathbb{R}$ are still valid on networks. For instance, the smoothing estimate for the linear equation with constant coefficients is still valid. Although its classical proof on $\mathbb{R}$ relies on Fourier analysis, one may easily adapt the proof in [6] which uses only integrations by parts and Besovs spaces that can still be defined on a tree using the heat operator. Strichartz estimates has been used previously to treat controllability issues for the NSE in [27]. The possible applications of the present results in the control context remains to be analyzed. We mention here some previous works on the controllability/stabilization of the wave equation on networks [12, 31.

Finally, another problem of interest is the study of the dispersion properties for the magnetic operators analyzed in [23, [21. The analysis in this case is more difficult since in the presence of an external magnetic field the effect of the topology of the graph becomes more pronounced. In contrast with the analysis done here, in the case of magnetic operators the graphs are viewed as structures in the three dimensional Euclidean space $\mathbb{R}^{3}$ and the orientation of the edges becomes important.

\section{REFERENCES}

[1] S. Alexander. Superconductivity of networks. A percolation approach to the effects of disorder. Phys. Rev. B (3), 27(3):1541-1557, 1983.

[2] F. Ali Mehmeti. Nonlinear waves in networks, volume 80 of Mathematical Research. Akademie-Verlag, Berlin, 1994.

[3] V. Banica. Dispersion and Strichartz inequalities for Schrödinger equations with singular coefficients. SIAM J. Math. Anal., 35(4):868-883, 2003.

[4] J. von Below. Classical solvability of linear parabolic equations on networks. J. Differential Equations, 72(2):316-337, 1988.

[5] J. L. Bona and R. C. Cascaval. Nonlinear dispersive waves on trees. Can. Appl. Math. Q., 16(1):1-18, 2008.

[6] N. Burq and F. Planchon. Smoothing and dispersive estimates for 1d Schrödinger equations with BV coefficients and applications. J. Funct. Anal., 236(1):265-298, 2006.

[7] R. H. Cameron, Analytic functions of absolutely convergent generalized trigonometric sums, Duke Math. J. 3:682-688, 1937.

[8] J. P. Carini, J. T. Londergan, D. P. Murdock, D. Trinkle and C. S. Young. Bound states in waveguides and bent quantum wires. I. Applications to waveguide systems, Phys. Rev. B, 55:9842-9851, 1997.

[9] R. C. Cascaval and C. T. Hunter. Linear and nonlinear Schrödinger equations on simple networks. Libertas Math., 30:85-98, 2010.

[10] T. Cazenave. Semilinear Schrödinger equations. Courant Lecture Notes in Mathematics 10. Providence, RI: American Mathematical Society (AMS); New York, NY: Courant Institute of Mathematical Sciences. xiii , 2003.

[11] C. Cattaneo. The spectrum of the continuous Laplacian on a graph. Monatsh. Math., 124(3):215-235, 1997.

[12] R. Dáger and E. Zuazua. Wave propagation, observation and control in 1-d flexible multistructures, volume 50 of Mathématiques $\&$ Applications (Berlin) [Mathematics 8 Applications]. Springer-Verlag, Berlin, 2006.

[13] P. Exner. Vertex coupling in quantum graphs: approximations by scaled Schroedinger operators, to appear in Proceedings of the ICM satellite conference "Mathematics in Science and Technology" New Delhi, August 15-17, 2010.

[14] A. Figotin and Y. A. Godin. Spectral properties of thin-film photonic crystals. SIAM J. Appl. Math., 61(6):1959-1979 (electronic), 2001.

[15] I.M. Gelfand, D.A. Raikov and G.E. Chilov, Les anneaux normés commutatifs, Monographies internationales de mathématiques modernes, Gauthier-Villars, Paris, 1964.

[16] J. Ginibre and G. Velo. The global Cauchy problem for the nonlinear Schrödinger equation revisited. Ann. Inst. H. Poincaré Anal. Non Linéaire, 2(4):309-327, 1985. 
[17] S. Gnutzmann and U. Smilansky. Quantum graphs: Applications to quantum chaos and universal spectral statistics. Advances in Physics, 55(5-6):527-625, 2006.

[18] L.I. Ignat, Strichartz estimates for the Schrdinger equation on a tree and applications, SIAM J. Math. Anal., 42(5):2041-2057, 2010.

[19] V. Kostrykin, J. Potthoff, and Robert Schrader. Contraction semigroups on metric graphs. In Analysis on graphs and its applications, volume 77 of Proc. Sympos. Pure Math., pages 423-458. Amer. Math. Soc., Providence, RI, 2008.

[20] V. Kostrykin and R. Schrader. Kirchhoff's rule for quantum wires. J. Phys. A, 32(4):595630, 1999.

[21] V. Kostrykin and R. Schrader. Quantum wires with magnetic fluxes. Comm. Math. Phys., 237(1-2):161-179, 2003.

[22] V. Kostrykin and R. Schrader. Laplacians on metric graphs: eigenvalues, resolvents and semigroups. In Quantum graphs and their applications, volume 415 of Contemp. Math., pages 201-225. Amer. Math. Soc., Providence, RI, 2006.

[23] P. Kuchment. Graph models for waves in thin structures. Waves Random Media, 12(4):R1-R24, 2002

[24] P. Kuchment. Quantum graphs. I. Some basic structures. Waves Random Media, 14(1):S107-S128, 2004. Special section on quantum graphs.

[25] P. Kuchment. Quantum graphs: an introduction and a brief survey. In Analysis on graphs and its applications, volume 77 of Proc. Sympos. Pure Math., pages 291-312. Amer. Math. Soc., Providence, RI, 2008.

[26] P. Kuchment and O. Post. On the spectra of carbon nano-structures. Comm. Math. Phys., 275(3):805-826, 2007.

[27] L. Rosier and B. Y. Zhang. Exact boundary controllability of the nonlinear Schrödinger equation. J. Differential Equations, 246(10):4129-4153, 2009.

[28] Z. Sobirov, D. Matrasulov, K. Sabirov, S. Sawada, and K. Nakamura. Integrable nonlinear schrdinger equation on simple networks: Connection formula at vertices. PHYSICAL REVIEW E, 81, 2010

[29] R.S. Strichartz. Restrictions of Fourier transforms to quadratic surfaces and decay of solutions of wave equations, Duke Math. J. 44(3):705-714, 1977.

[30] T. Tao. Nonlinear dispersive equations, volume 106 of CBMS Regional Conference Series in Mathematics. Published for the Conference Board of the Mathematical Sciences, Washington, DC, 2006. Local and global analysis.

[31] J. Valein and E. Zuazua. Stabilization of the wave equation on 1-D networks. SIAM J. Control Optim., 48(4):2771-2797, 2009.

(V. Banica) Département de Mathématiques, Université D’Evry, Bd. F. Mitterrand, 91025 Evry, FrancE

E-mail address: Valeria.Banica@univ-evry.fr

(L. I. Ignat) Institute of Mathematics "Simion Stoilow" of the Romanian Academy, 21 Calea Grivitei Street, 010702 Bucharest, Romania

AND

BCAM - Basque Center for Applied Mathematics,, Bizkaia Technology Park, BuildING 500 Derio, BAsque Country, Spain.

E-mail address: liviu.ignat@gmail.com 